\title{
EM BUSCA DE CONHECIMENTO DE ENFERMAGEM SOBRE 0 HOMEM COM CÂNCER: UMA EXPERIÊNCIA INTERNACIONAL
}

\author{
Searching for a nursing knowledge about the man with \\ cancer: an international experience

\section{En búsqueda del conocimiento de enfermería sobre el hombre con cáncer: una experiencia internacional}

\section{RESUMO}

Trata-se de um relato de experiência de visita técnico-científica e intercâmbio cultural na Escola de Enfermagem - Universidade da Califórnia de Los Angeles, no período de janeiro a junho de 2008, durante o desenvolvimento do curso de mestrado na Escola de Enfermagem Anna Nery da Universidade Federal do Rio de Janeiro. 0 propósito é apresentar as atividades desenvolvidas em disciplinas e grupo de pesquisa. A experiência contribuiu para o aprofundamento de aspectos teóricos relacionados à temática do homem portador de câncer e às possibilidades de intervenção de enfermagem. Sugere-se que estratégias institucionais sejam implementadas pelos programas de pós-graduação, com o apoio dos órgãos de fomento para viabilizar experiências de intercâmbio internacional durante o curso de mestrado, pois favorecem grande avanço pessoal e profissional, além de estreitar laços de cooperação com pesquisadores e instituições do exterior para o desenvolvimento de pesquisas no seguimento da formação profissional.

Palavras-chave: Intercâmbio Educacional Internacional. Educação de Pós-Graduação em Enfermagem . Enfermagem Oncológica.

\begin{abstract}
This study presents an experience report about an international exchange in the School of Nursing - University of California Los Angeles in the period of January - June 2008, during the development of the Master Program at Anna Nery Nursing School - Federal University of Rio de Janeiro. The purpose is to present the activities developed during this period, in courses done and participation in a group of research. The experience contributed to improve theoretical aspects related to our research that involves men with cancer and nursing management. It is suggested that institutional strategies should be implemented by the Graduate Programs, with a support by the government financial agencies to offer international experiences of international, exchanged during the Master Program. These international experiences provide a deep impact on personal and professional development. The international exchange is also an intervention to facilitate an international partnership.
\end{abstract}

Keywords:International Educational Exchange, Graduate in Nursing, Oncology Nursing.

\section{Resumen}

Se trata de un relato de experiencia de visita técnico-científica e intercambio cultural en la Escuela de Enfermería de la Universidad de California, Los Ángeles, entre enero y junio de 2008 durante el desarrollo del Curso de Maestría en la Escuela de Enfermería Anna Nery de la Universidad Federal de Rio de Janeiro. El objetivo es presentar las actividades desarrolladas en asignaturas y grupo de pesquisa. La experiencia fue útil para la profundización de aspectos teóricos relacionados a la temática del hombre portador de cáncer y las posibilidades de intervención de enfermería. Se sugiere la implementación de estrategias institucionales por parte de los programas de postgraduación, con el apoyo de los organismos de fomento para tornar viables las experiencias de intercambio internacional durante el curso de maestría, puesto que favorecen grandes avances personales y profesionales, además de estrechar la cooperación con investigadores e instituciones extranjeras para el desarrollo de pesquisas en el área de la formación profesional.

Palabras clave: Intercambio Educacional Internacional. Educación de Postgraduación en Enfermería. Enfermería Oncológica.

'Enfermeira. Mestranda da Escola de Enfermagem Anna Nery/UFRJ - Núcleo de Pesquisa Gestão em Saúde e Exercício Profissional da Enfermagem. Membro do Grupo de Pesquisa Gerência e Processo de Cuidar na Enfermagem em Oncologia. Brasil. E-mail: mariagefe@gmail.com, ${ }^{2}$ Enfermeira. Doutora em Enfermagem. Professora Adjunta da Escola de Enfermagem Anna Nery/UFRJ - Núcleo de Pesquisa Gestão em Saúde e Exercício Profissional da Enfermagem. Coordenadora do Grupo de Pesquisa Gerência e Processo de Cuidar na Enfermagem em Oncologia. Brasil. E-mail: marleachagas@gmail.com, ${ }^{3} \mathrm{PhD}, \mathrm{RN}$, Assistant Professor UCLA School of Nursing. USA. E-mail: smaliski@sonnet.ucla.edu 


\section{INTRODUÇÃO}

A formação de recursos humanos qualificados para suprir a demanda do mercado de trabalho especializado, além de cientistas e pesquisadores que contribuíssem para 0 desenvolvimento econômico e científico do país, foi motivação para o desenvolvimento da pós-graduação no Brasil na década de 60 do século passado. Instituíam-se as diretrizes para uma política científica e tecnológica nacional, sob coordenação do atual Conselho Nacional de Desenvolvimento Científico e Tecnológico(CNPq) e a Coordenação de Aperfeiçoamento de Pessoal de Nível Superior (CAPES).

Assim, desde a década de 70 do século passado, os enfermeiros têm envidado esforços para se integrar nessa política governamental. 0 expressivo crescimento da pósgraduação na Enfermagem brasileira a partir dos anos de 1980, com a criação de cursos de doutorado, a formulação de estratégias para expansão dos cursos e redução das desigualdades regionais para acesso aos programas, além dos avanços na inserção de representantes da Enfermagem no CNPq e na CAPES sinalizam o potencial da Enfermagem para ocupar o seu lugar e definir suas particularidades enquanto área de conhecimento ${ }^{1,2}$.

E no investimento coletivo, para situar a Enfermagem no campo da ciência, vários desafios precisam ser superados, entre eles a internacionalização. Tal desafio se configura como uma das diretrizes gerais do Plano Nacional de Pós-Graduação (2005-2010) e deve ser implementado através de políticas de cooperação internacional e de formação de recursos humanos no exterior ${ }^{3}$. Dessa forma, considerando o atual contexto de formação de recursos humanos em nível stricto sensu, os intercâmbios internacionais se constituem estratégias institucionais que contribuem para o fortalecimento dos programas de pós-graduação em consonância com as diretrizes da política pública.

Nessa linha de pensamento, este relato tem o objetivo de apresentar as atividades desenvolvidas durante visita técnicocientífica e intercâmbio cultural na School of Nursing University of California, Los Angeles, no período de janeiro a junho de 2008.

A motivação para realização desta atividade se fundamenta na necessidade de aprofundamento de aspectos teóricos relacionados à dimensão organizacional do cuidado de enfermagem ao homem portador de câncer diante das suas necessidades de ajuda; tema da pesquisa em andamento como projeto de dissertação do Curso de Mestrado da Escola de Enfermagem Anna Nery, da Universidade Federal do Rio de Janeiro.

Tal necessidade se justificou durante a realização do estado da arte para elaboração do projeto quando se verificou que, no âmbito nacional, as publicações referentes a essa temática ainda são incipientes. Utilizando como fontes de dados a produção científica da última década que focalizasse o homem com câncer, pôde-se constatar que a saúde do homem não tem sido objeto de estudo na maioria dos estudos. As publicações identificadas foram produzidas principalmente nas áreas de urologia e epidemiologia, onde o enfoque eram aspectos clínicos, terapêuticos e epidemiológicos do câncer. Nas pesquisas específicas da Enfermagem, questões relativas ao gênero masculino emergem na discussão dos resultados de estudos que contemplaram clientes de ambos os sexos.

Já no âmbito internacional, utilizando a base de pesquisa PUBMED, localizou-se um grupo de pesquisadores na University of California, Los Angeles (UCLA) cujas publicações nos remeteu ao projeto de dissertação em andamento. Trata-se do UCLA's Jonsson Comprehensive Cancer Center, um grupo formado por cerca de 240 pesquisadores envolvidos em pesquisas que tratam da prevenção, detecção, controle, tratamento e educação na área de oncologia. Como integrantes do grupo destacam-se a enfermeira e professora da School of Nursing/UCLA, Dra. Sally Maliski, e colaboradores que no período de 2002 a 2007 publicaram 17 artigos na nossa área de interesse. Dessas produções, a quase totalidade, 16 , refere-se à pesquisa e um artigo de revisão. Quanto ao enquadramento metodológico, todos os estudos utilizam o método qualitativo. As temáticas focalizaram, em quase todos (15), o homem como objeto de estudo; uma pesquisa tratou de uma revisão sobre pesquisas na área da enfermagem oncológica e outra tratou da experiência de sobrevivência ao câncer de pulmão.

Sendo assim, estabelecemos contato com a Dra. Maliski para conhecer seu trabalho e ampliar o conhecimento sobre a temática de estudo, visando ao melhor delineamento da pesquisa. Além disso, vislumbrou-se a oportunidade de aprimoramento técnico-científico em uma instituição internacional.

Após aceite da instituição, foi elaborado um roteiro de visita técnica para guiar o diário de campo onde foram registradas as atividades desenvolvidas, observações e, principalmente, as reflexões acerca das vivências em seu contexto temporalespacial.

\section{ATIVIDADES DESENVOLVIDAS DURANTE A VISITA}

\section{Participação em Disciplinas do Curso de Pós- Graduação da UCLA}

0 primeiro passo da visita foi integrar-se ao grupo de pesquisa da Dra. Maliski, que, no período da visita, era a responsável por três disciplinas do curso de Pós-Graduação da School of Nursing/UCLA (uma para o curso de mestrado e duas para o curso de doutorado). 0 convite para participar das aulas representou uma excelente oportunidade de integração e aprendizado.

A disciplina para o curso de mestrado intitulava-se "Human Responses To Cancer" e objetivava discutir a aplicação de teorias/pesquisas à prática clínica, enfatizando a avaliação e a intervenção de enfermagem em resposta ao câncer. 0 enfoque 
era o cuidado de enfermagem na prevenção, rastreamento, diagnóstico, tratamento, manejo de sintomas, reabilitação e qualidade de vida de pessoas com câncer.

Nessa disciplina, as aulas eram ministradas pela professora Maliski e por enfermeiras convidadas que trabalhavam diretamente com a temática em questão. Eram abordados diversos aspectos que abrangia desde a epidemiologia até novos tratamentos em teste, com ênfase no planejamento e na implementação do cuidado de enfermagem. Eram estimuladas as trocas de experiências entre os estudantes, que, em sua maioria, já trabalhavam como enfermeiros na área de oncologia. Em um segundo momento da disciplina, os estudantes apresentavam seminários referentes ao manejo de sintomas mais comuns que os pacientes com câncer costumam apresentar, como náuseas e vômitos, caquexia, fadiga, dispneia, neutropenia-pancitopenia, dor, ansiedade-depressão, disfunção sexual, entre outros.

As disciplinas cursadas com os doutorandos eram relacionadas à abordagem teórico-metodológica de estudos qualitativos. Uma das disciplinas, intitulada "Advanced Qualitative Research Methodology l", destinava-se a estudantes do segundo ano do curso que pretendiam desenvolver estudos de natureza qualitativa, utilizando a Grounded Theory como referencial metodológico. 0 conteúdo programático da disciplina contemplava uma proposta de desenho da pesquisa, incluindo estratégias para seleção de sujeitos, técnicas de entrevista para coleta de dados e conhecimentos básicos para análise de resultados com base no referencial enunciado.

As aulas, proferidas pela professora Maliski, eram teóricopráticas e apresentavam dois momentos distintos, um de discussão teórica, baseado em leituras pré-estabelecidas, e outro, de prática, no qual realizávamos a apresentação das entrevistas realizadas como atividades extraclasse. Nesse momento, eram discutidas técnicas de entrevista, além de introdução sobre o início do processo de análise de dados.

A outra disciplina do curso de doutorado cursada intitulavase "Advanced Qualitative Research Methodology II" e tratavase da continuação da disciplina anterior com enfoque na análise dos dados produzidos a partir da entrevista. Esta disciplina foi proferida pela professora MarySue Heilemann, que também utiliza a metodologia qualitativa no desenvolvimento de suas pesquisas.

As aulas objetivavam desenvolver nas estudantes habilidades para realização do processo de análise de dados qualitativos utilizando técnicas diversas e, para tal, também contava com dois momentos distintos: o de discussão teórica de textos e de prática, com o debate das entrevistas que eram realizadas pelas doutorandas como atividade extraclasse. Caso já estivessem em fase de coleta de dados para sua pesquisa e possuíssem autorização do comitê de ética, as entrevistas debatidas já eram as mesmas que compunham a amostra de suas teses. 0 processo começava ao serem lidas e discutidas as entrevistas; assim eram visualizados dados "crus" para iniciar o processo de análise, linha a linha, identificando significância, regularidade e similaridade de dados. Posteriormente, tais dados davam origem a categorias, e, para auxiliar a elaboração e organização destas, foram apresentadas técnicas para construção de mapas, matrizes e diagramas que auxiliassem na caracterização e dimensionamento das mesmas.

Outra atividade proposta pela disciplina era a realização de memos que consistiam em reflexões analíticas de nossos pensamentos. Os memos deveriam ser realizados de maneira informal, estabelecendo uma conversa de você com você mesmo sobre seus dados, podendo incluir aprofundamentos teóricos, intuições e possíveis relações entre eles.

\section{UCLA's J onsson Comprehensive Cancer Center}

Esse centro de atenção a pessoas com câncer, criado na década de 60 do século XX por um grupo de cientistas e voluntários da UCLA, é composto, atualmente, por cerca de 240 pesquisadores envolvidos em pesquisas que tratam da prevenção, deteç̧ão, controle, tratamento e educação na área de oncologia. Caracteriza-se como um grupo transdisciplinar que tem o compromisso de desenvolver pesquisas no contexto do tratamento e apoio a pacientes com câncer.

Integrante desse grupo, a professora Sally Maliski desenvolve sua pesquisa focando o homem com câncer de próstata. Em sua linha de pesquisa, procura entender como esse homem vivencia o câncer diante das alterações provocadas em sua vida. Tratando destas temáticas, utiliza a abordagem qualitativa como referencial metodológico, pois entende que seu objeto de estudo trata de questões humanas e nãomensuráveis que requerem o aprofundamento e abrangência da compreensão na totalidade de suas múltiplas dimensões.

Como repercussão do seu trabalho, a professora Sally foi convidada a integrar a equipe de um programa chamado IMPACT - Improving Access Counseling and Treatment for Californians with Prostate Cancer (Melhorando o Acesso, Aconselhamento e Tratamento aos Californianos com Câncer de Próstata), um programa pioneiro desenvolvido pelo governo da Califórnia para fornecer acesso gratuito ao diagnóstico, tratamento e aconselhamento a homens com a referida patologia e que não têm condições de pagar o tratamento. A professora atua no programa como pesquisadora e gerente de enfermagem visando assegurar aos participantes apoio para enfrentamento das dificuldades, educação para saúde, ajuda para encontrar um médico de referência, cuidados específicos e apoio à família, numa perspectiva holística.

A experiência de pesquisa e organização da assistência de enfermagem da Dra. Sally com os homens com câncer de próstata que participam do referido programa possibilitou algumas reflexões. Existe hesitação dos homens californianos em procurar os serviços de saúde para atividades de prevenção e manutenção de saúde. Assim, como observamos no Brasil, os homens procuram os serviços de saúde quando percebem que há algo errado. Para a pesquisadora, tal fato se dá por ainda 
vivermos em uma sociedade patriarcal que acredita que as mulheres são para serem cuidadas e os homens dão o suporte, provêm e são fortes.

As leituras de livros e artigos científicos que eram posteriormente discutidos sobre a problemática em questão, $\mathrm{e}$ que enfocam as questões de gênero e cultura, além de participação da etapa de análise de dados de sua investigação atual, nos fez vislumbrar a possibilidade de colaboração entre nossos grupos de pesquisa a fim de investigar o fenômeno no interesse da organização da assistência de enfermagem.

\section{CONSIDERAÇÕES FINAIS}

A participação nas disciplinas possibilitou aprimoramento técnico-científico; ampliação de bases para compreensão do objeto de estudo e conceitos inter-relacionados; contribuição para enriquecimento metodológico do projeto de dissertação e realização de aprofundamento bibliográfico internacional. Outro importante ganho foi a troca de experiência com as colegas de classe uma vez que as disciplinas estimulavam a leitura e a discussão dos seus projetos, e, como cada uma se encontrava em uma determinada fase específica, as conversas funcionavam como incentivo e ajuda.

As discussões com Dra. Maliski acerca das investigações em andamento sobre homens com câncer de próstata e as leituras sobre a temática, a partir de revisão de literatura, contribuíram para aprofundar o conhecimento na área. Isso porque existe uma lacuna no conhecimento produzido acerca da temática relativa ao homem com câncer. Estudos recentes indicam que a produção científica da Enfermagem brasileira na oncologia focaliza as áreas de assistência e de organização do cuidado, principalmente referente às mulheres com câncer de mama e colo de útero, além de situações clínicas em que não há distinção do gênero dos participantes dos estudos ${ }^{4,5}$.

\section{REFERÊNCIAS}

1- Gutiérrez MGR, Vieira TT, Almeida MCP, Elsen I, Stefanelli MC. Acompanhamento e avaliação da pós-graduação no Brasil: retrospectiva histórica da representação da enfermagem. Esc Anna Nery Rev Enferm 2001 ago; 5 (2): 161-172.

2- Erdman AL, Mendes IAC, Leite JL. A enfermagem como área de conhecimento no CNPq: resgate histórico da representação da área. Esc Anna Nery Rev Enferm 2007 mar; 11 (1): 118-26.

3- Ministério da Educação (BR) Plano Nacional de Pós-GraduaçãoPNPG 2005-2010. Brasília (DF): CAPES; 2005.

\section{Agradecimentos}

Gostaríamos de agradecer à School of Nursing - University of California, Los Angeles, pela receptividade e atenção dispensada. Agradecemos às professoras Sally Maliski e MarySue Heilemann por possibilitarem a participação nas disciplinas stricto sensu, conferindo avaliação de participação, o que configurou a sua efetiva inserção.

Não poderíamos deixar de agradecer às enfermeiras estudantes dos programas de mestrado e doutorado das disciplinas cursadas por compartilharem suas pesquisas e nos acolherem nas aulas.
Sugere-se que estratégias institucionais sejam implementadas pelos programas de pós-graduação, com o apoio dos órgãos de fomento para viabilizar experiências de intercâmbio internacional durante o curso de mestrado, pois favorecem grande avanço pessoal e profissional, além de estreitar laços de cooperação com pesquisadores e instituições do exterior para o desenvolvimento de pesquisas no seguimento da formação profissional. Isso é possível através da concessão de bolsas de estudo no exterior, cujo programa oferece a oportunidade de realização de estágio apenas a estudantes regularmente matriculados em cursos de doutorado.

Contudo, é preciso planejar outras estratégias de intercâmbio de estudantes. A relevância de tal experiência, na percepção de quem já a vivenciou, é que possibilita "atravessar as fronteiras do Brasil para melhor apreender os desafios que se impõem para as mudanças urgentes..." para ousar propostas que permitam " (re)construir novas práticas no campo da assistência, do ensino e da pesquisa, conjugando arte e ciência e transformando a própria enfermagem na ação de se (re)fazer, (re)pensar e (re)produzir no âmbito da saúde e da vida" : 6:362.

Os ganhos desta visita foram além dos seus objetivos, a relevância de tal experiência, na percepção de quem a vivenciou, é que esta foi uma oportunidade ímpar, de grande valor profissional e pessoal para aprimoramento.

Vale ressaltar que foram criadas oportunidades para 0 estabelecimento de futuras parcerias entre os grupos de pesquisa da School of Nursing - University of California, Los Angeles, e Escola de Enfermagem Anna Nery/UFRJ para o representa um ganho importante para a comunidade científica e da Enfermagem brasileira.

4- Moreira MC, Camargo TC, Carvalho V, Figueiredo CF, Rosa LD, Bolzan MF. A pesquisa na área da enfermagem oncológica: um estudo das publicações em periódicos nacionais. Texto Contexto Enferm 2006 out/dez; 15(4): 595-600.

5- Silveira CS, Zago MMF. Pesquisa brasileira em enfermagem oncológica: uma revisão integrativa. Rev Latino-am Enfermagem 2006 jul/ago; 14(4):614-19.

6- Souza KV. Intercâmbio educacional internacional na modalidade doutorado sanduíche em enfermagem: relato de experiência. Esc Anna Nery Rev Enferm 2008 jun; 12 (2): 358 - 63. desenvolvimento de um projeto em colaboração, o que 